\title{
Rapidly Prototyping Marker Based Tangible User Interfaces
}

\author{
Maribeth Gandy ${ }^{1}$, Brian Jones ${ }^{1}$, Scott Robertson ${ }^{1}$, \\ Tiffany O`Quinn', and Amos Johnson² \\ ${ }^{1}$ Interactive Media Technology Center, Georgia Institute of Technology \\ \{maribeth, brian, scott, tiffany\} @imtc.gatech. edu \\ ${ }^{2}$ Computer Science Department, Morehouse College \\ ajohnson@morehouse.edu
}

\begin{abstract}
Tangible user interfaces (TUIs) can create engaging and useful interactive systems. However, along with the power of these interfaces comes challenges; they are often so specialized and novel that building a TUI system involves working at a low level with custom hardware and software. As a result the community of people that are capable of creating TUIs is limited. With this project we aim to make a particular class of TUIs accessible to a broader range of designers and HCI researchers by exposing TUI specific tools in a mixedreality rapid prototyping environment know as DART (The Designer's Augmented Reality Toolkit). In this paper we discuss the creation of a system for rapidly prototyping marker based tangible user interfaces. These prototyping tools were then used to create a set of TUI-based applications with the goal of raising students' interest in science via an exploration of fine art concepts.
\end{abstract}

Keywords: Tangible User Interfaces, rapid prototyping, mixed reality, toolkits.

\section{Introduction}

Tangible User Interfaces (TUIs) are a promising avenue for HCI research and a diverse array of TUI projects have been developed over the past fifteen years [6]. However, along with the unique power of these interfaces comes a drawback; they are often so specialized and novel that building a TUI system often involves working from scratch with custom hardware and software. As a result the community of people that are capable of creating TUIs is limited and the ability to iterate, test, and deploy them is also restricted. There has been considerable interest in the development of TUI toolkits that aid in the rapid prototyping and deployment of TUI systems [9], and there is still need for work in this domain. In particular there is a need for toolkits that enable TUI development by people without access to specialized hardware or low level programming expertise.

In this project we aim to make a particular class of TUIs accessible to a broader range of designers and HCI researchers by exposing TUI specific tools in a mixedreality (MR) rapid prototyping environment know as DART (The Designer's Augmented Reality Toolkit). DART exposes a variety of tracking and MR prototyping services (e.g. "Wizard of Oz" support) that can be incorporated in standard Adobe Director applications; thus providing a mature rapid prototyping environment for 
augmented reality (AR). This tool provided a starting point for our work which was to identify the additional components that were needed to support the creation of TUIs with DART and to incorporate them into a toolkit we refer to as DART-TUI. In this paper we discuss the creation of this system for rapidly prototyping marker based tangible user interfaces. The resulting tool (DART-TUI) was then used as part of an initiative to create novel and engaging interactive experiences for students with the goal of increasing their interest in science via an exploration of fine art concepts.

In the following sections we will discuss further the need for this type of tool and the underlying architecture of DART. We will examine the similarities and differences between the needs for AR and TUI prototyping tools. Then we will discuss in detail the additional components we created for DART that supports TUI development. Lastly we will discuss the first application we have created with our toolkit; a suite of art-themed applications that let students use tangible interfaces to have a constructivist learning experience to explore scientific concepts such as optics and fractals.

\section{Background}

Many unique and inspired TUIs have been developed over the years; ranging from a marble based music composition tool [2], to Topobo, a robot construction kit [13], to Designer's Outpost which allows web designers to layout sites via post-it notes [8], to a physical system for developing multimedia narratives [11]. As TUIs these systems are defined by the fact that the user sends input and (possibly) receives output through physical objects. Therefore a key component of a TUI system is the ability to track various types of objects such as marbles [2], drinking glasses [16] and ping pong balls [7]. In the systems mentioned above the tracking is often achieved via RF tags placed on the objects [11] (or magnetic switches [13)] and surfaces that are capable of sensing them. Computer vision [8] and acoustics [7] based systems are also used. While these approaches provide a robust and unobtrusive solution, working with such technologies requires both expertise and resources that may not be available to everyone wanting to experiment with TUIs and the resulting systems are hard to duplicate and move.

However for over a decade AR researchers have been utilizing an open source toolkit that uses paper based markers for tracking, called the ARToolkit [5]. The ARToolkit can also be used as the method of tracking tangible objects in a TUI. Of course, there are drawbacks to the use of visual markers for this task. The markers must remain in view of the camera and the user may have a hard time detecting when they are not. A user's hand occluding part of the marker will stop the object from being tracked. Fast movement of the markers will affect the tracking as will light levels. There are limitations on the range of motion (particularly rotation) for an object since the entire marker must always be in view. Lastly having to place a relatively large marker on an object can detract from its aesthetic qualities and its affordances for manipulation. However, based on our experience with our science education application we feel that these drawbacks can be accounted for in the design of the TUI and 
that the advantages (inexpensive, easy to deploy and replicate) outweigh these disadvantages. Therefore in DART-TUI we leverage this simple tracking mechanism, which can be used for cheap and easy prototyping.

From the beginning, researchers have designed TUIs that utilize the tabletop paradigm. From Wellner's DigitalDesk [15] to table top tangible games such as fish pong [16], many TUI systems have been based on the user interacting with objects placed on a table-top surface. While TUIs can come in other forms (like Topobo) the tabletop interface is a common one and is amenable to the marker based tracking we were leveraging in DART-TUI .Therefore our components are specifically crafted for this table-top TUI approach.

This is not the first project to tackle the concept of TUI prototyping. For years researchers working in the TUI domain have recognized the need for prototyping tools. In fact DART was first discussed as a platform for TUI extensions at Pervasive Computing 2004 [3]. There is not room in this paper to catalog the variety of projects that have looked at the taxonomy of tangible interactions [14], user definable TUIs [12], and hardware prototyping [4] for example. However one toolkit is of particular interest in the context of DART-TUI. Klemmer's Papier-Mache is a tool with some similarities to DART but originally created with TUIs in mind [9]. Papier-Mache provides rapid prototyping services including Wizard of $\mathrm{Oz}$ functionality and supports an array of tracking and identification technologies. And the designer is able to swap out technologies throughout the design process (e.g. prototyping with bar codes and deployment with RFID).

\section{DART}

DART is a set of tools added onto Adobe (formerly Macromedia) Director that allows designers and non-technologists to rapidly prototype augmented reality (AR) applications [10]. DART (and DART-TUI ) applications are created with "Actors" which are any sort of asset in the application (e.g. 3D object, audio clip, billboarded texture etc.) or physical object the user is interacting with. These Actors can be linked directly or indirectly to "Trackers" which are visual markers, a VRPN device, or a phidget sensor. "Cues" are used to generate events triggered by occurrences such as the appearance of a visual marker, two Actors within a specified proximity, the pressing of a button etc. "Actions" are what happen as a result of Cues (e.g. when a marker comes into view an audio clip is played or a virtual Actor becomes visible in the scene). In section 5 we will discuss how DART-TUI utilizes modified versions of these basic components for TUI specific functionality.

All the behaviors that are provided with Director (and are part of DART) are written in Lingo and editable by the user. Therefore a common approach is for developers to modify the standard behaviors while also writing their own from scratch. This is key to understanding the design of DART-TUI. We have created a suite of TUI specific scripts, but some of them were crafted for the specific needs of our initial application. This is not a shortcoming of the toolkit but rather is the standard way of working with Director. We provide a set of tools that may be useful "out of the box" to TUI designers however these scripts can be easily modified or built upon. In fact this process of building up a library of TUI components is an effective way of 
providing TUI tools. As discussed previously TUIs come in many different forms and it would be impossible to anticipate every component a TUI designer would need. The attempt to do so would either result in an overly complicated generalized system or one that is only of use in a particular type of application. Instead DART-TUI can evolve over time based on the needs of the user.

\section{Requirements: AR vs. TUI}

There are many similarities between the requirements of AR and TUI applications. Both require some sort of tracking technology. In AR applications the goal is to track the position of the user's eye so that virtual 3D graphics can be rendered such that they are properly registered with the physical world [1]. In AR applications that use marker tracking the goal is usually to register 3D content at the location of the markers. In TUIs the tracking needs are more focused on monitoring the position and orientation of the tangible objects the user is interacting with rather than the user's head/eye. While all the AR specific functionality of DART intended for head tracking and registering 3D graphics is still available to TUI developers our goal in with the DART-TUI additions was to provide components that fit with the "trackable objects" paradigm and make it easy for TUI developers to define the relationships between object location and application input. By using marker based tracking of objects this tool then allows designers to rapidly prototype a TUI by simply printing out a few markers and attaching them to the object they wish to track. The system then uses a webcam to track their positions. Of course this system restricts the types of TUIs that can be prototyped, but still supports a wide variety of interfaces. And marker tracking can be switched out for more sophisticated sensors in the DART-TUI application at any time.

In AR applications the output and rendering are typically focused on presenting 3D graphics overlaid on the live video stream. The display modalities and types of content presentation in TUIs are much more varied. Therefore DART-TUI decouples the tracking and presentation of content and allows the developer to more easily map tracker location to functions such as the movement of a $2 \mathrm{D}$ pointer.

Similarly since registration is often the goal in AR there are limits on the types of modifications you can make to tracker data that would impact the ability of the system to tightly link the graphics to physical objects. TUIs however do not have this constraint. Therefore the DART-TUI components let the designer apply transformations to the data such as smoothing so as to make the interactions more fluid for the user.

\section{The DART-TUI Toolkit}

Our prototyping system supports the creation of TUIs that have two main components: physical objects that will be manipulated by the user and a work area in which the user will interact with the objects. Contained within DART-TUI are behaviors that allow the designer to define the work area, the objects, and the application's response to object manipulation. 
The first step in designing a TUI is defining the workspace in which a user will interact. One approach for defining a space is to use a book paradigm. The pages can have text, illustrations, and other guides printed on them but these pages also act as the trigger to expose a particular activity or interaction set to the user. Therefore we created the "BookTracker" behavior which is used to create a workspace that consists of one or more "pages"; each page has printed on it a unique marker. With the BookTracker it is up to the designer to define what the cue generated by the appearance of a page will trigger. In our applications we use the cue to advance the application to a new exercise. An advantage of using the book workspace is that since we wanted to create applications that could be replicated and moved (e.g. our science education app was to be deployed in various student computer labs) this approach lets us define a workspace without delicate calibration or setup. When using the BookTracker the designer can have all the other trackers in the application report their position and orientation relative to the page marker, therefore the correspondence between movements of the objects and input to the system will remain constant no matter the angle, position, or distance of the page relative to the webcam. The other advantage of the book workspace is that it supports natural navigation through an experience (e.g. flipping to another page to choose another exercise, moving back and forth between pages to review previous information) and allows for content physically printed on the page to enhance the tangible feel of the experience and supports the users' interactions with the objects.

The "TableTracker" is a behavior that is used to track any number of physical objects within the work space. The TableTracker has all the capabilities of the original "LiveTracker" from DART (see [10] for more detail), but with some TUI specific features. First, as mentioned previously, the coordinates of the physical objects are reported relative to the marker on the work area page so that the designer can rely on the objects reporting within a set range of values regardless of how the book/work area is placed under the camera. Unlike AR applications, TUI designers may often wish to ignore parts of the tracker information so as to limit the degrees of freedom of the physical objects. In our science education application it was common that for a particular activity we would simply need the position of an object; using rotation data only made the interaction more confusing to the user. Therefore the TableTracker lets the designer indicate which degrees of freedom she wishes to be reported by the system. The tracker data can also be passed through a filter component(s). Marker tracking often results in constant small pose estimate errors that manifest as jittering of the registered objects in AR. This jittering was less acceptable in a TUI where movements of the objects were transformed and then used to control various types of 2D and 3D interfaces. Therefore we created a "smooth" component that minimized these tracker errors. This type of filtering would be unacceptable in an AR system where the result would be a loss of registration between the marker and the $3 \mathrm{D}$ object, but it was very useful for TUI development. These filters are simply treated as trackers themselves that consume live tracker data and then pass it on to subscribers transparently. It is therefore possible to combine several filters together seamlessly. We created "smooth" and "delay" trackers to modify our TUI tracker data, but developers can easily create their own filters by using these basic scripts as templates. 
For TUI control, the designer needs additional tools to transform the raw tracking information into meaningful control data for the application; the interface or application that is ultimately affected by the user's manipulation of the tangible objects does not have the natural correspondence between marker pose and virtual object rendering that an AR application has (in fact our science application uses both $2 \mathrm{D}$ and $3 \mathrm{D}$ environments as the output). In DART-TUI once the physical object information has been filtered it can be passed on to a variety of "controllers" that are fed into the interface of the designer's choice. It was important to build them with an architecture that makes it easy for designers to create controllers of their own since as previously discussed a characteristic of TUIs is the unique mappings between object interaction and application response. For example, in our application we created controllers that would transform the movement of objects into a change in camera field-of-view in a $3 \mathrm{D}$ scene, navigation of the virtual camera through a 3D scene, movement of a $2 \mathrm{D}$ pointer, rotation of a 3D object, and intensity of a light in a 3D scene among others.

Due to the structure of DART it is quite easy for a designer to add their own controllers to the existing set. A controller simply subscribes to a tracker (which may in fact be a filtered version of a live tracker) and, if needed, applies a "tracker specific transform" which maps the coordinates reported by the tracker into the coordinate system appropriate for this controller's function (e.g. a controller that controls the movement of a $2 \mathrm{D}$ point on the screen projects the reported points into $2 \mathrm{D}$ screen coordinates). The controller then has a set of properties related to its specific function. For example the Camera Controller that lets the user dolly a camera in a 3D scene asks the designer for the amount of time it should take the virtual camera to reach the next point. The controller will then interpolate between the current camera position and the new position so as to further smooth the movement in the world and to make the camera movements pleasing even if the user picks up a marker and moves it to another location. There is also a property that specifies whether the camera should always point at the origin or simply maintain its original orientation. The Rotation Controller which is used to rotate a $3 \mathrm{D}$ object lets the designer specify which actor to apply the rotation to and $\mathrm{min} / \mathrm{max}$ angles of rotation for the 3 axes.

\section{The STEM Application}

Our first application created with DART-TUI presents students with a suite of activities which aim to teach them about science topics via art lessons (e.g. light, perspective, and fractal math). This work was sponsored via an NSF project led by Morehouse College that is studying the efficacy of using simulation environments that include artistic elements to enhance Science, Technology, Engineering and Mathematics (STEM) curricula at the high school level. An emphasis on creativity and passion was intended as an antidote to negative student attitudes about these fields.

To use the STEM application the student sits in front of a desktop computer; instead of a keyboard or mouse there is a physical book in front of her and a webcam is placed on the monitor such that is has a view of the book from above. The student accesses the activities by paging through the physical book (see Fig 1.). Each page contains printed illustrations and instructions to guide and enhance the activity. The student places various plastic paddles on the book page to interact in the activity. To 


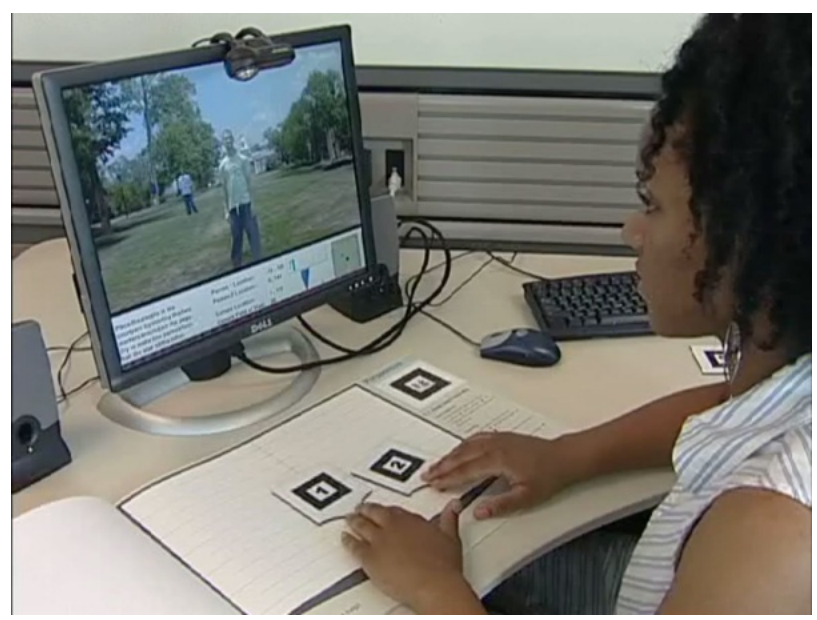

Fig. 1. A student interacts with an exercise in the Perspective module

get guidance with the system the student places a paddle with a "?" on the page it to access the help system. The TUI system allowed us to create an interface for the activities utilizing two handed interactions and direct manipulation. The student can experience a variety of activities such as subtractive color mixing, changing lighting effects in a still life, and using magic lenses to see paintings in new ways.

There are several advantages of this TUI approach for the exercises. In general we wanted to provide a novel and fun interface which would make these activities more engaging to the students than a typical desktop computer keyboard and mouse based application. Also, in the exercises the students are often manipulating multiple variables simultaneously (e.g. coordinated dollying and zooming of the virtual camera, generating seed values and iterations of a fractal) which is afforded by the two handed control of the TUI. The tangible interface also gives subtle and expressive control over the input parameters (e.g. color mixing). Since we are teaching via art it is fitting that the students have a sense of applying their own creativity (e.g. fractal creation, lighting a still life, camera effects etc.) to the experience. The TUI also allows us to convey spatial concepts through the interface (e.g. the student can understand the ratios of apparent size to distance from the viewer in the perspective module because she can see the relative distance the two paddles are from each other on the page). This tangible approach also allows us to leverage physical media such as the book pages (e.g. putting information, guides, and other content on the physical book pages). Lastly, the result is a natural interface that supports exploration (e.g. turn to another book page to go to another section, turn back and forth as you please, put the "?" paddle on the page to get help). The students can discover through experimentation how the tangible objects (paddles) affect the activity (e.g. multiple paddles defining fractal creation and magic lenses). 


\subsection{Application Interactions}

In this section we will highlight a few of the thirteen exercises from three modules that make up the STEM application and discuss the tangible interfaces for each.

In the Perspective module the student does a variety of tasks that help illustrate the concept of perspective, how field of view of a camera affects our perception of it, and how depth is conveyed in 2D art via vanishing points. The first set of exercises take place in a 3D scene created from photographs of the Morehouse campus (not unlike a diorama) (See Fig. 1). In the fourth exercise in this module two paddles are used to change the camera location and the FOV in this scene. The student is challenged to recreate the "zoom in/ dolly out" style effect that is seen in movies such as "Vertigo" and "Jaws." Sliding paddle 1 vertically on the page will change the FOV while doing the same motion with paddle 2 will dolly the camera in and out. The mappings are defined such that moving the paddles in opposite directions will achieve the desired effect (e.g. "zooming in" by reducing FOV while simultaneously dollying the camera out).

The Light Module allows students to explore the concept of light, its importance to art and our perception of it. In one exercise the student is exposed to how the human eye works via a series of "magic lenses" that they can move over a painting via the paddles. First the student moves a lens over Seurat's "Sunday in the Park". The area under the lens is magnified. This allows the student to see how individual dots of different colors are perceived at a distance as a mixture of those colors. Then the student manipulates a series of lenses over a second painting (each paddle represents a different type of lens). Through the lenses she can see how a person with a vision impairment experiences a scene (color blindness, retinitis pigmentosa etc.)

In the Fractal module the student is able to create and modify fractals and learns how they appear in nature as well as art. In the first exercise the student creates an iterated function system (a method of generating fractals). Two paddles define a starting position and orientation of two block figures in 2D. The third paddle dynamically increases and decreases the number of recursive iterations of function generator. In another the students are exposed to fractals in art (architecture). Ba Ila villages in Zambia have a pseudo fractal pattern. The student builds her own Ba Ila village by placing huts in the scene (paddle 1 is used to move a hut around the world and when paddle 2 is placed on the page the hut is dropped) and a virtual village is generated around these starting conditions. The student can then browse their village via paddle 1 which is used to rotate the $3 \mathrm{D}$ scene.

\section{Conclusion and Future Work}

Through this process of design and implementation we have identified the components that are necessary to repurpose an AR design tool for use as a TUI prototyping system. In particular these components allow the designer to define a workspace, and map object position/orientation to arbitrary functions in the application. The DARTTUI toolkit is particularly useful for rapidly creating inexpensive and portable tabletop TUI prototypes. A designer can download the code, print out the markers, and then begin working with her own TUI environment. We have utilized our toolkit 
to create an educational system (the STEM application) that uses tangible interactions and fine-art concepts to expose students to science topics.

This STEM education application is in the process of being evaluated by Morehouse College researchers with high school students. In the future we plan to add additional exercises and to gather data on the usability and learning effects of our system. DART (with the DART-TUI components) is available for download and we are eager to see our toolkit applied to additional TUI applications.

\section{Acknowledgements}

The authors would like to acknowledge the students at Morehouse College who helped create the content for the STEM application. Also a special thanks to the all developers of DART over the years which include Prof. Blair MacIntyre who lead the original project and to Steven Dow who contributed significantly to its design and implementation. This work was funded by NSF Grant \#0625731

\section{References}

1. Azuma, R.T.: A Survey of Augmented Reality. Presence: Teleoperators and Virtual Environments 6(4), 355-385 (1997)

2. Bean, A., Siddiqi, S., Chowdhury, A., Whited, B., Shaer, O., Jacob, R.J.: Marble track audio manipulator (MTAM): a tangible user interface for audio composition. In: Proceedings of the 2nd international Conference on Tangible and Embedded interaction, Bonn, Germany, February 18-20 (2008)

3. Gandy, M., Dow, S., MacIntyre, B.: Prototyping Applications with Tangible User Interfaces in DART,The Designer's Augmented Reality Toolkit. In: Toolkit Support for Interaction in the Physical World Workshop at IEEE Pervasive Computing 2004, April 20 (2004) (positional paper)

4. Greenberg, S., Fitchett, C.: Phidgets: easy development of physical interfaces through physical widgets. In: Proceedings of the 14th Annual ACM Symposium on User interface Software and Technology, Orlando, Florida, November 11-14 (2001)

5. Kato, H., Billinghurst, M.: Marker Tracking and HMD Calibration for a Video-Based Augmented Reality Conferencing System. In: Proceedings of the 2nd IEEE and ACM international Workshop on Augmented Reality, October 20-21 (1999)

6. Ishii, H., Ullmer, B.: Tangible bits: towards seamless interfaces between people, bits and atoms. In: Proceedings of the SIGCHI Conference on Human Factors in Computing Systems, Atlanta, Georgia, United States, March 22-27 (1997)

7. Ishii, H., Wisneski, C., Orbanes, J., Chun, B., Paradiso, J.: PingPongPlus: design of an athletic-tangible interface for computer-supported cooperative play. In: Proceedings of the SIGCHI Conference on Human Factors in Computing Systems: the CHI Is the Limit, Pittsburgh, Pennsylvania, United States, May 15-20 (1999)

8. Klemmer, S.R., Newman, M.W., Farrell, R., Bilezikjian, M., Landay, J.A.: The designers' outpost: a tangible interface for collaborative web site. In: Proceedings of the 14th Annual ACM Symposium on User interface Software and Technology, Orlando, Florida, November 11-14 (2001) 
9. Klemmer, S.R., Li, J., Lin, J., Landay, J.A.: Papier-Mache: toolkit support for tangible input. In: Proceedings of the SIGCHI Conference on Human Factors in Computing Systems, Vienna, Austria, April 24-29 (2004)

10. MacIntyre, B., Gandy, M., Bolter, J., Dow, S., Hannigan, B.: DART: The Designer's Augmented Reality Toolkit. In: Proceedings of the 2nd IEEE/ACM international Symposium on Mixed and Augmented Reality, October 7-10 (2003)

11. Mazalek, A., Davenport, G., Ishii, H.: Tangible viewpoints: a physical approach to multimedia stories. In: Proceedings of the Tenth ACM international Conference on Multimedia, Juan-les-Pins, France, December 1-6 (2002)

12. Mugellini, E., Rubegni, E., Gerardi, S., Khaled, O.A.: Using personal objects as tangible interfaces for memory recollection and sharing. In: Proceedings of the 1 st international Conference on Tangible and Embedded interaction, Baton Rouge, Louisiana, February 1517 (2007)

13. Raffle, H.S., Parkes, A.J., Ishii, H.: Topobo: a constructive assembly system with kinetic memory. In: Proceedings of the SIGCHI Conference on Human Factors in Computing Systems, CHI 2004, Vienna, Austria, April 24-29 (2004)

14. Shaer, O., Leland, N., Calvillo-Gamez, E.H., Jacob, R.J.: The TAC paradigm: specifying tangible user interfaces. Personal Ubiquitous Comput. 8(5), 359-369 (2004)

15. Underkoffler, J., Ishii, H.: Urp: a luminous-tangible workbench for urban planning and design. In: Proceedings of the SIGCHI Conference on Human Factors in Computing Systems: the CHI Is the Limit, Pittsburgh, Pennsylvania, United States, May 15-20 (1999)

16. Wellner, P.: The DigitalDesk calculator: tangible manipulation on a desk top display. In: Proceedings of the 4th Annual ACM Symposium on User interface Software and Technology, Hilton Head, South Carolina, United States, November 11-13 (1991)

17. Yoon, J., Oishi, J., Nawyn, J., Kobayashi, K., Gupta, N.: FishPong: encouraging humanto-human interaction in informal social environments. In: Proceedings of the 2004 ACM Conference on Computer Supported Cooperative Work, Chicago, Illinois, USA, November 6-10 (2004) 